arrangement he then traces on the outer surface of the evelid in the two portions separated by the natural fold of the skin, the upper portion constituting about onethird of the surface of the lid, the lower the remaining two-thirds. From these he makes the pathological de. duction, that certain forms of disease within the orbit that are seated in, or internal to, the motor apparatusthat is in the substance of, or within the cavity of the ocular tunic, extend their effects to the lower portion of the eyelid, with which they are continuous, and that cer. tain other affections situated external to the motor ap. paratus-which is that part of the orbit containing the fat-will show themselves by inflammation or other changes in the upper division of the same.

The last subject to be noticed in connection with the ocular tunic, is the greater light that Dr. O'Ferrall has been able to throw on the causes that protrude the eyeball. Inflammation of the ocular tunic will in itself cause ocular prominence. This is chiefly through effusion into the cellular membrane connecting it to the ball of the eye. The infiltration is apparent where the conjunctiva is reflected from the eyelid to the eyeball, just where this membrane closes up the ocular tunic in front. Here it receives the pressure of the effused serum, and becomes separated from the sclerotic coat by the extension of the infiltration. The amber coloured chemosis, originating in inflammation of the conjunc. tiva, is distinguished from it by redness as well as serous infiltration.

\section{ON THE USE OF NITRIC ACID IN THE CURE OF ULCERATION OF THE OS AND CERVIX UTERI.}

By Hammett Haing, M.R.C.S.Eng., formerly HouseSurgecn to the Milland Counties Lying.in Hospital.

At a meeting in 1862 of one of the leading London medical societies, the subject of discussion was the value of nitrate of silver as an escharotic in cases of ulceration of the os and cervix uteri. Having for years past had extensive experience in the treatment of uterine disease, and tried the various kinds of applica. tions in general use, both fluid and solid, and finding but one upon which I could rely in producing perma. nent amelioration of the disease, viz., pure nitric acid,I will select from my notebook a f'ew well-marked cases of ulceration in its various stages, from the phagedænic down to simple excoriation, that have not only been cured, but bave borne inspection some years afterwards, and have never in a single instance in my practice shown a tendency to return-a fact that cannot be induced in favour of nitrate of silver, or any other solid, or, 1 believe, fluid, caustic application now in general use.

My nomenclature of diseases affecting the os and cervix uteri is, like the treatment, simple: 1 . Cases of cancerous ulceration, or disease with apparent malig. nant tendency, where the ulcer is large, excoriated, dis. charging pus or matter of a mucopurulent character, in conjunction with great derangement of the general health-in fact, every symptom of cachexia; 2 . Cases of simple ulceration or excoriation, with or without hypertrophy, accompanied by symptoms not quite so distress. ing to the general health. I will detail a few cases of each variety; and then offer some remarks on the rela tive value of the various applications, solid and fluid, generally employed in the treatment of ulceration situ. ated about the mouth of the uterus. Of the first variety, or cases of an apparent malignant nature, I have selected three examples.

CASE. In the year 1850 , I was requested by a gentleman to visit a poor woman aged 42 , the mother of six children, the youngest two years old. Since the birth of this child she dated her failing health.
For the last fourteen months she had been gradually losing flesh, with pains in the back, sickness, loss of appetite, perspirations, enstipation, and slight cough. She had been treated for pulmonary disease by a private practitioner, and had undergone a digital uterine examination in a neighbouring infirmary. On examination with the speculum (May ith), I found the uterus prolapsed, and the os imbedded in a mass of mucopurulent discharge (although the patient used daily alum injections), on the removal of which both labia of the os uteri were found extensively ulcerated some distance up the cervix. The patient was directed to have a liberal diet, and for medicine a vegetable tonic with iodide of potassium; for local application, the nitrate of silver. At the end of two months, finding my patient no better and the ulceration but little checked, I resolved to change my treatment both locally and generally. Having washed the parts ulcerated with a moist sponge, and freed the sore from all discharge. I painted the dis. eased portion with pure nitric acid, requesting the patient to keep the speculum (one of Fergusson's) fixed in its present condition for half an hour. The application caused no pain of importance. Internally, I gave twenty minims of the tincture of sesquichloride of iron twice a day, and left her for a fortnight. At the expiration of this period, I ngain applied the speculum, and was surprised at the improvement that had taken place. The excavated portions of the ulcer were quite filled up, and the whole disease presented a most healthy appearance. The same treatment was persisted in, and the nitric acid was again painted over the unhealed portion of the ulcer. From this time the symptoms gradually im. proved; the discharge of mucopurulent matter decreased; and, in less than two months from the time when the nitric acid was first applied, the patient's bealth was quite restored.

In the year 185:3, I attended her in labour of her seventh child. There was not a single symptom of uterine mischief present; nor did there appear any cicatrix to interfere with the proper contraction and dilatation of the os uteri. 'This patient died of consumption in 1858 , without presenting another indication of uterine disease.

CASE Ir. In the year 1859 , M. H., a poor woman, aged 33 , sent for me one night, having been suddenly seized with a pain over the region of the heart and fainting. She was a washerwoman, and had been delivered of an illegitimate child three years before. She considered her disease to be of two years standing.

There was great emaciation; vomiting after meals ; cough ; loss of appetite; diarrhora; fainting upon the slightest exertion or excitement; and every symptom of general cachexia. The catamenia were regular, but attended with pain and difficulty in micturition. Between the menstrual periods, the discharge was exces. sive, thick, and grumous. On examination wilh the speculum, the vagina appeared red and inflamed, and gave evidence of its dis(r)ered condition by frequent small discharges of blood. The os uteri, as in the former case, was encased by a layer of mucopurulent matter, on the removal of which the following state of disease presented itself. The labia of the os could not be defined; but the whole cervix appeared one mass of ulcerated disease ( so far as a bivalve speculum could dis. play), bleeding upon the slightest touch. The men. strual period being over, nitric acid was applied; the ulcerated parts being well covered with it, by means of a glass brush, as before. A pledget of wool was then introduced, fastened to a piece of silk, and passed up the vagina, so as to cover the diseased portion. This application was repeated three times in six werks; and the last examination displayed a sound and heallby looking surface. The medical treatment was the same as in the last case-tonies, with a generous diet. The patient has remained quite well un to the present; the 
catamenia being regular, and no discharge occurring in the interval. 'This patient had been twice in an in firmary within the last year, but had only been examined digitally; and lotiuns of zinc and lead had been injected.

CASE IIr. In April 1860, I saw in consultation M. $\mathrm{H}$., of a consumptive family, the mother of three children, the youngest fourteen months old. She had been ill for twelve months, and confined to her bed during five. She was much emaciated; the countenance was pale; breathing short; pulse feeble. She had occasional attacks of hysteria with violent palpitation, accompanied with pulsation of the descending aorta suffi. cient to raise the bedclothes. The catamenia were regular; the bowels constipated. She had no pain over the region of the uterus, but occasionally a discharge of mucopurulent matter, accompanied with pain.

After a careful examination, no organic disease being discovered, I suggested an examination of the uterus with the speculum, which was complied with. After washing away the thick stratum of mucopurulent matter in which the os uteri was imbedded, I discovered a large excavated ulcer situated upon the posterior labia of the os, and extending up into the cervix. The ulcer was very extensive, and evidently of the sloughing variety. I applied a good layer of the pure nitric acid, retaining the sneculum until every chance of the adjacent mucous membrane being touched was over; and, on its removal, I plugged the vagina with wool. A tonic with hydrocyanic acid was given internally, to allay the violent sickness which disturbed her during the day; and generous diet with port wine was ordered. Ten days from this date, I again applied the speculum, and found the ulcer nearly healed, and the general symptoms much improved. The nitric acid was again applied to the parts of the ulcer not already cicatrised, and the general treatment continued. The patient slowly re. covered her strength; the ulcer became perfectly healed, without another application; the discharge became natural; and in the early part of 1862 she was again delivered of a fine child, without the slightest indication of uterine disease being present.

I will now give some cases of simple ulceration of the os and cervix uteri, with or without hypertrophy.

CASE IV. In the year 1855 , a lady, aged 30 , married, consulted me for relief from a disagreeable taste in the mouth, sickness after taking food, lumbar pain, accom. panied by severe constipation. She had never borne children, but had miscarried twice, and considered she was now three months advanced in the family way. I prescribed rest and the remedies usual in such cases, but without success; for she aborted the following week. In about six weeks after this event, when my patient had quite recovered, I requested to be allowed an ex. amination with the speculum, which was granted. Upon examination, there appeared a slight amount of hypertrophy, for both labia of the os were elevated; but the fissure, quite into the cavity, was ulcerated and slightly excavated, looking as if small pieces had been cut out, discharging a fluid, not mucopuriform, as in the former cases, but such as would be expected from an ulcer on any other portion of the body. The whole of the dis. eased surface was fully displayed by a Fergusson's speculum; therefore, without removing the instrument, I freely painted the diseased portions with nitric acid with a glass brush, as before, allowing the liquid to pass into the cavity; requesting the patient to retain the speculum in statu quo for half an hour-this being the most effectual method of preventing the acid from injuring the adjacent parts. I next prescribed for her twenty drops of the syrup of the iodide of iron and manganese twice a day, and a liberal diet. I found it necessary to apply the acid to the surface ulcerated in about ten days, after which period, with the exception of slight hypertrophy, the part was quite healed, and the patient paid her usual annual visit to the sea. I have since attended her in two accouchements; and the mother and both children are alive and well.

CASE Y. C. S., aged 2i, married, consulted me in 1850. When single, she had suffered from dysmenorrhoea, and between the menstrual periods from a discharge of a grumous character. Since her marriage, she had miscarried twice-once at six months, and the other time at the fourth month. She had all the appearance of being enceinte. The papilla on the breasts were formed. I supposed her to be about four months advanced. I recommended rest; but in a few days afterwards I received a letter to say she had miscarried, and was going on well. Six wetks after this event, I requested an examination with the speculum, which was granted. The os uteri appeared swollen; but, on close inspection, the orifice between the labia presented an ulcerated appearance, with little indura. tions on its surface. The nitric acid was allowed to touch the whole of this surface; and this operation was repeated before the next menstrual period. The tinc ture of the sesquichloride of iron was given internally, and liberal diet and moderate exercise in the open air were recommended. Her recovery was rapid. I have since delivered her of two children-one in 1858, the other in 1860 . The old symptoms have not shown any tendency to return.

$\mathrm{CASE}_{\mathrm{S} E}$ v. In the year 1858 , I visited Mrs. B., aged 20 , married, the mother of one child. She had suffered since her confinement from dyspepsia, with pains in the back, occasional sickness, palpitations, and extreme nervous irritability. She had taken tonics, and had also consulted a London practitioner, who had applied the nitrate of silver, with temporary advantage.

On examination with the speculum, the os uteri externally appeared much hypertrophied; but the whole of the internal surface of the labia was much excoriated, exuding a muco puriform discharge. The nitric acid was applied as on the former occasion, being allowed to pass into the neck as far as possible. The tonic treatment was continued; and, one month afterwards, not the slightest urevenness or ulcerated surface remained. The hypertrophy continued for some time, causing painful defrecation; but, with the aid of sea air and bathing, it ultimately disappeared.

REvarks. If, in the language of Dr. R. Lee, the women of England have suffered from the injudicious use of the speculum matricis, I think it is our duty to occasion. ally record cases containing proofs of the benefit result. ing from its judicious employment. I am quite aware there are men even in the present day, well versed in uterine pathology, who believe and freely assert that for all practical purposes, digital examination is sufficient to explore all the ordinary affections of the os and cervix uteri. The gentleman quoted above says, "I am fully satisfied that the speculum does not enable us to decide earlier than the finger that cancer has commenced; and if it did so, as some maintain, and enable us to make applications to the os uteri, which could not be made without, not the slightest advantage would be gained in practice."

With regard to the first part of this statement, I have no wish to dispute the powers which some persons possess, from extensive experience, of ascertaining or obtaining a knowledge of diseases by the organ of touch but as to the latter part, viz., the inutility of appropriate applications in the ulcerative stage of uterine diseases, including malignant, I wholly differ. Hundreds of females have testified to the comfort and freedom from pain experienced from the use of judicious applications to disease of the uterus, even in its worst stage-the process of ulceration. What, for instance, would have been the fate of Case No. II, where the poor woman had for months undergone digital examination, and consumed gallons of injection of lead and alum; and where, though 
reduced to a shadow and despairing of ever receiving relief, after the loss of the greater part of the os and cervix uteri by ulceration, she recovers strength and is restored to her original health and vigour, and at the present time pursuing the most arduous of female occupations?

There is one remark emanating from the pen of Dr. R. Lee, viz., the impropriety of using the speculum to the virgin uterus, with which I quite coincide. For my own part, I never make use of it in the unmarried; and there is a certain class of cases of young females, with fair hair and light complexions, of the strumous diathesis, who suffer much from dysmenorrhæa when single, become much worse after marriage, and frequently miscarry. Upon examination with the speculum, ulceration, with or without hypertrophy, will, in almost every instance, be found the cause of mischief; and, in a mild degree, was no doubt the cause of the dysmenorrhæa prior to marriage. It is a question in my mind which would be the most correct course of practice; to cure this malady prior to marriage, and thus save the patient much suffering mentally and bodily, or to wait until the mischief has taken place, before the remedy is applied.

The subject under discussion, not being one treating of the diagnosis or exploration of uterine mischief, but relating to the properties of the various applications now in use for the amelioration or cure of that stage of in flammation of the most accessible portion, called ulcera tion, I will, in describing these applications, divide them into two classes; viz., solid and fluid. The first, or solid, are the lunar caustic; potassa fusa ; the actual cautery; and lastly, Mr. Ellis's electric cautery. The second class, or fluid applications, are, injections of various sub. stances, such as solutions of lead, alum, oak bark infusion, nitrate of silver, and various other substances, according to the nature of the case ; and for escharotics we have chloride of antimony, tincture of the sesquichloride of iron, the acid nitrate of mercury, and the various mineral acids,

Before dilating upon the merits of these various applications, we must first see what we require them to do for the diseased parts, in order to bring about the object desired; and to do this correctly, I cannot do better than quote a remark of Dr. Bennet on the subject. "The only treatment to be depended upon, is one of such stimulation of the ulcerated part as to modify its vitality in such a manner as to induce a healthy action, and finally cicatrisation." Taking this as our text, and the above named substances as our pharmacopœia, we must next consider upon which to pin our faith.

The first amongst the solids, the nitrate of silver, has been for years past the favourite in general practice, and has in many instances succeeded; but it is very uncer. tain and requires constant repetition, very much to the annoyance of the patient and to the dissatisfaction of the surgeon. The result arrived at, from extensive practice private and public, is that it is only palliative, and I am convinced that if the opinion of the majority of the profession who have used the remedy were taken, they would coincide with my verdict.

The second on the list, potassa fusa, may be associated with the actual cautery, from neither of which I have seen much benefit derived, but often great constitutional irritation. With regard to the electric-cautery, all I can say is, that women have already a great repugnance to the use of the speculum, and in all probability the addition of an electric-battery in that delicate region would cause a sensation of still greater disgust. The fluids, I think, give us more hope for success; considering, that to cure effectually an ulceration of any magnitude, the application must not only be made to the ulcerated surface, but must dip into the cavity itself.

The first on this list-injections-may be passed over, as requiring but few remarks. Their use is vaginal only; for, in nineteen cases out of twenty, they never reach the os uteri; therefore, except in cases of leucorrhœa, or cancer, as cleansers of the vagina, or to convey some temporary relief in the form of an opiate, they are useless.

The next remedy to that which I am about to extol, is the acid ritrate of mercury, an application highly spoken of by Dr. Bennet. I believe that it possesses great stimulant and healing properties; but, from my experience of its use, it is not equal to the pure nitric acid. What the former accomplishes in two or three applications, the latter will perform in one.

My attention was directed to the powerful escharotic and healing powers of nitric acid some years past, in a case of deep-seated syphilitic ulceration of the perinæum, when every other remedy had failed. It not only destroyed the diseased membrane, but at the same time produced a healthy action in the new tissues-a change so rapidly accomplished that both patient and surgeon were singularly surprised by the sudden metamorphose. Of all the membranes in the body the mucous seems to be the most adapted for this remedy, as shewn by the extraordinary power which it possesses of stimulating and producing a healthy action when applied to the ulcerated mucous membrane in cases of internal piles. Very little irritation is produced by its application.

In cases of cancer of the uterus when the stage of ulceration has commenced, and hæmorrhage made its appearance, nitric acid will be found the safest and most styptic application in arresting its progress. The tincture of sesquichloride of iron will not be found sufficiently powerful in such cases as an external application; but, as an internal remedy, it performs its office more effectually.

In conclusion, after reviewing the few cases here recorded, and again adverting to my text-" Stimulation to produce a healthy action, finally cicatrisation"-I may with safety say, there are three things necessary for the cure (not palliation) of ulceration of the os and cervix uteri ; first, a full view of the diseased surface; secondly, supporting the general health; thirdly, the application of an appropriate powerful escharotic, that will not only touch the external portion of the os uteri, but will dip full into its cavity ; the practitioner, armed with the tincture of sesquichloride of iron as an internal and the pure nitric acid as an external remedy, need never despair of not fulfilling these intentions.

I am quite aware that the plan adopted by me in the cases recorded is not novel, but employed freely by other practitioners; but, considering that confirmation of treatment is the essence-the principle-upon which the profession wish to rely, my cases are designed to confirm the nitric acid treatment in cases of ulceration of the os and cervix uteri; and with this view I am inclined to give my experience of the great value of the agent.

Testimonial. A tea-service has been presented to Mr. C. Parsons, by the nurses and patients of the North Staffordshire Infirmary, as a mark of the kindness which they received at his hands whilst House Surgeon of the Infirmary.

The King of the Belgrans. Professor Langenbeck's first visit to the King of the Belgians resulted in the greatest success. The stone was almost entirely removed. King Leopold, however, has thought it prudent to request the professor to defer his second visit for some time, as His Majesty fears his strength will be unequal to so speedy a repetition of the operation. Professor Langenbeck speaks with surprise of the clearness of mind and self.command displayed by the old king, who did not permit so painful an operation to prevent him from discharging his usual business. The distinguished surgeon received 200 Friedrich d'Or (£170) as honorarium for his services.

167 